\title{
Sales minerales en la ganadería de leche bovina
}

\section{Mineral salts in dairy farming cattle}

\author{
Pérez M A, Peña FA, y Benítez MJ1
}

${ }^{1}$ Estudiantes de Medicina Veterinaria y Zootecnia. Escuela de Ciencias Animales curso de profundización "Alternativas de alimentación en el Trópico"

Universidad de los Llanos

\section{mapp40@gmail.com}

\section{RESUMEN}

Los bovinos obtienen los minerales necesarios para su metabolismo del medio ambiente en el que se desarrollan. Las deficiencias y desbalances de minerales en la dieta son reconocidas como una de las limitantes en la producción animal. Los minerales cumplen un importante papel en la nutrición, teniendo en cuenta que aunque no proporcionan energía son esenciales para la utilización y síntesis biológica de nutrientes esenciales. En los sistemas intensivos que utilizan vacas de alta producción, la administración de sales minerales permite incrementar el consumo y por ende la productividad de los animales. Los minerales son diariamente utilizados por los animales en el desarrollo de sus funciones fisiológicas normales, y en determinadas ocasiones la falta de estos en la dieta normal del animal es la causa primaria de problemas reproductivos. La implementación de las dietas minerales depende de las deficiencias que presente el suelo y los pastos en los que se alimentan los animales. Sin embargo, la administración de sales mineralizadas de tipo comercial tiene que ser realizada bajo análisis técnicos los cuales arrojen como resultado la fórmula ideal para cada terreno. El presente artículo muestra algunas de las características a tener en cuenta en la alimentación de ganado de lechería en Colombia, partiendo de variables como el desarrollo fisiológico del animal, los requerimientos minerales de acuerdo a su estado de producción y las condiciones de los suelos en los que el animal pastorea.

Palabras clave: Requerimientos minerales, producción de leche.

\section{ABSTRACT}

Cattle get the minerals needed for metabolism of the environment in which they thrive. The deficiencies and imbalances of minerals in the diet are recognized 
as one of the limiting factors in animal production. Minerals play an important role in nutrition, taking into account that although not essential to provide energy use and biological synthesis of essential nutrients. In intensive systems using high-producing cows, the administration of mineral salts can increase consumption and hence the productivity of animals. Minerals are daily used by animals in the development of its normal physiological functions, and in some cases the lack of these in the animal normal diet is the primary cause of reproductive problems. The implementation of mineral diets depends on the efficiencies that this soil and pastures where animals are fed. However, the management of commercial mineral salts must be carried out under technical analysis which shed as a result the ideal formula for each field. This paper presents some of the features to consider in feeding dairy cattle in Colombia, based on variables such as the physiological development of the animal, mineral requirements according to their production status and soil conditions in the grazing animal.

Keywords: Mineral requirements, milk production.

\section{INTRODUCCIÓN}

Los bovinos reciben los minerales que requieren del medio ambiente los pastos, el agua y la tierra adherida a los forrajes que consumen. La mayor cantidad de minerales aprovechados por los bovinos llega entonces por el consumo de pastos y la tierra que esta adherida a ellos, por lo tanto debe considerarse que un buen manejo de la nutrición mineral consiste en aportar la cantidad necesaria según los requerimientos de la vaca, lo que puede ser factible de evaluar mediante su determinación en los diferentes tejidos del animal y saber así, si el aporte es suficiente o no (Ceballos et al., 2004). Diferentes investigaciones demuestran que en vacas lecheras del cruce Holstein x Cebú, son similares a las utilizadas para la producción lechera en Colombia (Otálora et al., 2009), las absorciones parciales de minerales sugieren que el retículo-rumen es el sitio más importante para el aprovechamiento de calcio $\left(\mathrm{Ca}^{++}\right)$y magnesio $\left(\mathrm{Mg}^{++}\right)$, mientras que en los intestinos delgado y grueso ocurriría lo propio con fosforo y potasio (Sandoval et al., 1998). Este factor demuestra que una absorción apropiada de minerales está directamente relacionada con el correcto funcionamiento del tracto 
digestivo del bovino, lo cual es controlable mediante el manejo de la alimentación, estimulando un funcionamiento normal a nivel ruminal.

Las deficiencias y desbalances de minerales en la dieta son reconocidas como una de las limitantes a la producción animal. Uno de los primeros pasos en la evaluación de la nutrición mineral del ganado en pastoreo, es la conducción de muestreos de suelos, forrajes y fluidos y tejidos animales a fin de analizar la concentración de los minerales de importancia nutricional (Balbuena et al., 2003).

Cada mineral se administra teniendo en cuenta la condición física en la que se encuentra el animal. Los requerimientos de calcio y fósforo dependen de la producción y composición de la leche, además del estado de preñez. Las vacas en producción requieren de niveles elevados de calcio en el alimento, mientras que para las secas suministrar un alto nivel de calcio tiene como consecuencia desfavorable una disminución de calcio en el suero sanguíneo (hipocalcemia), en el parto o cerca de él. La hipocalcemia está asociada con un aumento en la incidencia de mastitis, cetosis, desplazamiento de abomaso, retención de placenta y menor fertilidad (Gómez, 2008). Todo animal requiere de un constante equilibrio entre la cantidad de mineral que es consumido y aprovechado para el desarrollo de sus funciones metabólicas.

En el presente documento se recopila información de investigaciones estructuradas sobre la nutrición mineral del ganado de lechería en Colombia, partiendo de variables como el desarrollo fisiológico del animal, los requerimientos minerales de acuerdo a su estado de producción y las condiciones de los suelos en los que el animal pastorea.

\section{SITUACIÓN DE LA NUTRICIÓN EN BOVINOS DE LECHERÍA Y DE LAS SALES MINERALIZADAS EN COLOMBIA}

El suelo es un cuerpo natural conformado por una conexión de elementos y procesos, resultado de su localización y del contacto de la atmósfera con la superficie de la corteza; en ella se encuentran los silicatos, grupo amplio de minerales, producidos por las reacciones del silicio, el oxígeno y el aluminio, en cantidades que fluctúan alrededor del 80\% (Malagón, 2003). La calidad de los 
suelos depende de diferentes factores, el Instituto de la Calidad del Suelo (Soil Quality Institute, SQI) del Servicio de Conservación de Recursos Naturales (NRCS) del USDA (SQI, 1999), define la calidad del suelo como "la capacidad que tiene de llevar a cabo una serie de funciones básicas como mantener la productividad biológica, regular los flujos de agua y de solutos, amortiguar la contaminación y almacenar y circular nutrientes". Estos factores determinan el aporte que el suelo llega a hacer en el metabolismo del animal y de los indicadores de producción, partiendo de la calidad de los nutrientes consumidos convirtiéndose en una relación directa.

En Colombia se utiliza, oficialmente, el Sistema de Clasificación de Suelos del Departamento de Agricultura de los Estados Unidos de Norteamérica (Soil Survey Staff (SSS), 1999). Este sistema es multicategórico y agrupa los suelos, 12 clases que se llama "Orden" (Tabla 1), se describe el porcentaje de distribución de los diferentes órdenes según la región natural en la que se encuentran en Colombia. Las 12 clases de suelos definidas al nivel de orden, se identifican con los siguientes nombres: alfisol, andisol, aridisol, entisol, espodosol, gelisol, histosol, inceptisol, mollisol, oxisol, ultisol y vertisol. (Jaramillo, 2004).

Tabla 1. Distribución de los órdenes de suelos de Colombia, por regiones naturales

\begin{tabular}{|c|c|}
\hline REGIÓN NATURAL & ÓRDENES DE SUELOS DOMINANTES \\
\hline Amazonia & $\begin{array}{l}\text { Oxisol (36.9\%), Inceptisol (20.8\%), Ultisol (18.9\%), Entisol } \\
(16.1 \%) \text {, Espodosol (2.9\%) }\end{array}$ \\
\hline Andina & $\begin{array}{l}\text { Inceptisol (35\%), Entisol (33\%), Andisol (18\%), Alfisol (3\%), } \\
\text { Ultisol (3\%), Mollisol (2\%) }\end{array}$ \\
\hline Orinoquia & Oxisol (39\%), Inceptisol (28\%), Entisol (21\%), Ultisol (6\%) \\
\hline Llanura Caribe* & $\begin{array}{l}\text { Inceptisol (38\%), Entisol (31\%), Mollisol (6\%), Vertisol (6\%), } \\
\text { Aridisol (5\%), Alfisol (4\%), Oxisol (1\%) }\end{array}$ \\
\hline Andén Pacífico & $\begin{array}{l}\text { Inceptisol (50\%), Entisol (26\%), Oxisol (8\%), Ultisol (8\%), } \\
\text { Histosol (4\%) }\end{array}$ \\
\hline $\begin{array}{l}\text { Valles } \\
\text { interandinos }\end{array}$ & $\begin{array}{l}\text { Inceptisol (44.4\%), Entisol (40.1\%), Alfisol (3.7\%), Mollisol } \\
(3.7 \%) \text {, Vertisol (1.4\%), Andisol (0.8\%) }\end{array}$ \\
\hline Islas del Caribe & $\begin{array}{l}\text { Inceptisol (30\%), Entisol (22\%), Vertisol (15\%), Histosol (13\%), } \\
\text { Mollisol (12\%) }\end{array}$ \\
\hline
\end{tabular}

Para el caso de la Orinoquia Colombiana los elevados niveles de oxisoles ejercen efectos sobre la producción animal teniendo en cuenta las condiciones 
a las que están sometidas las pasturas. La vegetación nativa de la región comprende, en su mayor parte, gramíneas de escaso valor nutritivo (Álvarez y Láscano, 1987). Sus suelos, especialmente los oxisoles (Tropeptic Haplustox isohypertermic) (CIAT, 1978), tienen $\mathrm{pH}$ de 4.5 y baja disponibilidad de $\mathrm{Ca}, \mathrm{Mg}$, $\mathrm{K}$ y $\mathrm{P}$, además de una saturación de aluminio mayor de 80\% (Sanz y Seige, 1999), (Tabla 2). Estos factores los convierten en susceptibles a la degradación. En el caso de la utilización de estos suelos en las producciones ganaderas es necesaria la suplementación de los elementos minerales ajenos al suelo en la dieta del animal.

Tabla 2. Análisis de suelo de pradera con Brachiaria decumbens, utilizadas en el experimento de minerales ${ }^{1}$

\begin{tabular}{|c|c|c|c|c|c|c|c|c|c|c|c|c|}
\hline \multirow{2}{*}{ Pradera } & \multirow{2}{*}{ PH } & \multirow{2}{*}{$\begin{array}{l}\text { Mat } \\
\text { Org } \\
(\%)\end{array}$} & \multirow{2}{*}{$\underset{(p p m)}{P}$} & \multicolumn{5}{|c|}{$\begin{array}{c}\text { Miliequivalentes/100 gr de } \\
\text { suelo }\end{array}$} & \multicolumn{4}{|c|}{ Ppm } \\
\hline & & & & Al & $\mathrm{Ca}$ & Mg & $\mathrm{K}$ & $\mathrm{Na}$ & $\mathrm{Fe}$ & Mn & $\mathrm{Cu}$ & $\mathrm{Zn}$ \\
\hline 1 & 5,2 & 2 & 3 & 2,2 & 0,4 & 0,05 & 0,08 & 0,01 & 90 & 4,1 & 0,8 & 0,7 \\
\hline 2 & 5,0 & 2 & 2 & 2,0 & 0,1 & 0,04 & 0,08 & 0,01 & 80 & 5,6 & 0,6 & 0,5 \\
\hline 3 & 5,3 & 2 & 2 & 2,7 & 0,1 & 0,06 & 0,12 & 0,01 & 100 & 4,7 & 0,5 & 0,5 \\
\hline 4 & 5,2 & 2 & 3 & 2,1 & 0,3 & 0,05 & 0,10 & 0,1 & 100 & 4,5 & 0,6 & 0,5 \\
\hline
\end{tabular}

${ }^{1}$ Análisis realizados por el Laboratorio Nacional de Suelos de Tibaitatá. (Laredo et al., 1987).

El $82.88 \%$ del territorio colombiano se encuentra ubicado por debajo de los $1000 \mathrm{msnm}$ (clima caliente) y tiene una temperatura media mayor de $27^{\circ} \mathrm{C}$ (Malagón et al., 1995), Además, se ha estimado que aproximadamente el $80 \%$ del territorio presenta una condición climática húmeda, muy húmeda o pluvial (IGAC, 2003). Dichas condiciones climáticas han generado procesos intensos de alteración de los minerales primarios del material parental y de mineralización de la materia orgánica, así como de lavado (lixiviación) intenso de todo aquello que es soluble en agua (Jaramillo, 2004). Un aspecto práctico muy importante, consecuencia del modelo de evolución planteado para los suelos de clima cálido - húmedo, es la reducción del pH.

La máxima disponibilidad de los elementos que la planta consume en mayores cantidades del suelo: $\mathrm{N}, \mathrm{P}, \mathrm{K}, \mathrm{Ca}, \mathrm{Mg}, \mathrm{S}$, se presenta con valores de $\mathrm{pH}>6$. En los suelos fuertemente ácidos se da la máxima disponibilidad de los elementos menores: $\mathrm{Fe}, \mathrm{Mn}, \mathrm{Cu}, \mathrm{Zn}$ y B, los cuales pueden llegar a cantidades tóxicas con relativa facilidad en suelos con dicha condición de $\mathrm{pH}$, en el caso 
de la Orinoquia colombiana se han realizado análisis de suelo de las praderas, las cuales muestran concentraciones bajas en materia orgánica, fósforo y especialmente en los micro elementos cobre y zinc (Tabla 2) este factor se claramente reflejado en los niveles encontrados en los forrajes de la zona (Tabla 3) (Laredo et al., 1987).

Para entender los principales tipos de los suelos en Colombia hay que hacer relación a los elementos biofísicos que conforman las regiones naturales. Por ejemplo, en la Orinoquia altillanura son de alto grado evolutivo, poseen características asociadas con procesos de mayor alteración, lo cual genera menos aporte de elementos requeridos por las plantas y mayor dependencia nutritiva de su fracción orgánica, son suelos de ciclo largo donde predominan los óxidos de hierro y aluminio (IGAC, 2003). En cambio, las regiones de la Cordillera Andina tienen condiciones diferentes, muchas zonas han recibido aportes de cenizas volcánicas y por su ubicación en pendiente, presentan procesos erosivos y movimientos en masa, lo que afecta su estabilidad y desarrollo evolutivo. Por esta razón se consideran suelos más jóvenes y menos alterados (IGAC 2003 y Malagón, 2003).

Tabla 3. Concentración promedio mineral en pasto Brachiaria, durante el experimento. Base seca.

\begin{tabular}{ccccccccccc}
\hline \multirow{2}{*}{ Época } & \multicolumn{1}{c}{$\%$} & $\mathbf{c}$ & \multicolumn{4}{c}{ Ppm } \\
\cline { 2 - 11 } & $\mathbf{N}$ & $\mathbf{C a}$ & $\mathbf{P}$ & $\mathbf{M g}$ & $\mathbf{K}$ & $\mathbf{S}$ & $\mathbf{F e}$ & $\mathbf{M n}$ & $\mathbf{C u}$ & $\mathbf{Z n}$ \\
\hline Lluvia & 0,8 & 0,42 & 0,18 & 0,26 & 2,03 & 0,13 & 280 & 343 & 7 & 12 \\
Sequía & 0,6 & 0,53 & 0,18 & 0,26 & 3,06 & 0,13 & 565 & 515 & 7 & 18 \\
Valor Normal & 1,0 & 0,37 & 0,27 & 0,29 & 0,93 & 0,14 & 100 & 60 & 10 & 60 \\
\hline \multicolumn{2}{l}{ Modificado de Laredo et al., 1987.} & & & & & & &
\end{tabular}

LOS MINERALES EN LA ALIMENTACIÓN DE BOVINOS DE LECHERÍA

En muchos casos en fincas lecheras existen problemas de deficiencia sub-clínica de uno o más minerales, lo cual no es fácil de diagnosticar. (Foto, Marra LGGA RoA VGGA)

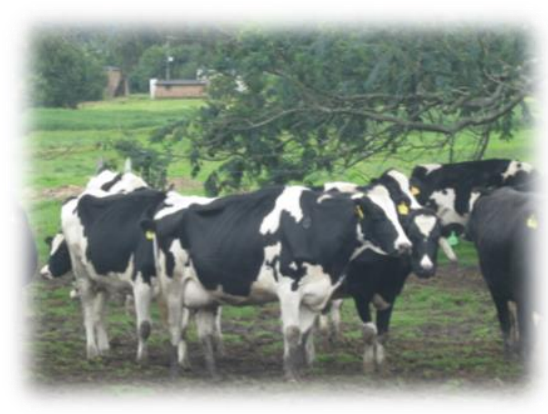


Los minerales cumplen un importante papel en la nutrición porque, aunque no proporcionan energía son esenciales para la utilización y síntesis biológica de nutrientes esenciales. En muchos establos lecheros existen problemas de deficiencia de uno o más elementos. Sin embargo, estos se presentan en forma sub-clínica la cual no es fácilmente diagnosticada. Este tipo de deficiencia podría causar pérdidas importantes en producción de leche debido a que los minerales cumplen un rol importante en la síntesis de leche, metabolismo y salud en general (Gómez y Fernández, 2009). Dependiendo la calidad de las pasturas utilizadas en la alimentación y el manejo y suplementación, se puede evitar que el animal sufra algún tipo de trastorno metabólico y se vea alterado su estado de producción. Se considera que un buen manejo de la nutrición mineral es saber cuánto de cada mineral necesita consumir dependiendo del estado fisiológico y cuánto es aportado por la ración, por lo que es importante conocer el contenido y biodisponibilidad de minerales de los diferentes alimentos que actualmente se utilizan en la preparación de las raciones.

Los bovinos son animales forrajeros por naturaleza, esto quiere decir que las pasturas son los alimentos con los que cubren todas sus necesidades clave mantenimiento, crecimiento, preñez y desarrollo corporal (Gómez y Fernández, 2009). En sistemas intensivos que utilizan vacas de alta producción, la suplementación permite incrementar el consumo y por ende la productividad de los animales en general; está demostrado que la suplementación disminuye el consumo de pradera, en especial cuando la disponibilidad de pradera es alta. (Balocchi et al., 2002). La administración de sales mineralizadas en la dieta diaria debe desarrollarse partiendo del resultado de los análisis de suelos que determinen la oferta y deficiencia de cada uno de los elementos en la pastura, buscando evitar posibles casos de intoxicaciones por excesos y disminuyendo los costos en la producción por desperdicio de sales.

Las fuentes de minerales se pueden clasificar en orgánicas e inorgánicas, dentro de las primeras pueden ser de origen animal como las harinas de carne, de hueso y de pescado, por otra parte, entre las fuentes vegetales los forrajes de alta calidad tienden a ser en general buenas fuentes de calcio ( $\mathrm{Ca}$ ) y relativamente bajos en fosforo $(P)$ en especial las leguminosas, no así algunos granos y subproductos (Vieyra, 2004). Por último, fuentes de minerales 
inorgánicas propiamente dichas como los fosfatos en el caso del $\mathrm{P}$, siendo los más utilizados ortofosfatos de calcio. De acuerdo a la cantidad de átomos de Ca en la molécula, se los clasifica en mono, di o tricálcico. La utilización de fosfatos alimenticios en las raciones permite gran flexibilidad para balancear la relación $\mathrm{Ca}$ : $\mathrm{P}$; una disponibilidad superior de $\mathrm{P}$; utilizar productos libres de posible contaminación microbiana y de malos olores, además contar con un aporte de P predecible y constante (Vieyra, 2004). La Tabla 4, muestra algunos de las principales fuentes de minerales de diferentes orígenes.

Tabla 4. Concentración de calcio y fósforo de distintas fuentes y su disponibilidad relativa

\begin{tabular}{clccc}
\hline BDR (\%) & \multicolumn{1}{c}{ Fuente de P } & Ca \% & P \% & Ca : P \\
\hline ALTA (120) & F. Monocálcico/ Monodicálcico & $15-18$ & $21-22$ & $0.7-0.8$ \\
MEDIA ALTA (100) & F. Dicálcico & 22 & 18 & 1.3 \\
MEDIA BAJA (<90) & Harina de huesos & 32 & 16 & 2.0 \\
& Harina de Pescado & 6 & 3.5 & 2.0 \\
& Alfalfa, Heno & 1.25 & 0.23 & 5.8 \\
& Alfalfa, Pasto. & 1.9 & 0.27 & $6-7$ \\
& Algodón, Semilla & 0.21 & 0.64 & 0.33 \\
& Gramíneas, Heno & 0.38 & 0.12 & 3.2 \\
& Maíz, Grano & 0.03 & 0.24 & 0.12 \\
& Maíz, Silaje & 0.20 & 0.19 & 1.0 \\
& Solla, Harina & 0.3 & 0.68 & 0.44 \\
& Sorgo, Grano & 0.04 & 0.34 & 0.12 \\
& Trigo & 0.13 & 0.9 & 0.13 \\
\hline
\end{tabular}

BDR: Biodisponibilidad Relativa. Modificado de Vieyra. (2004).

La deficiencia o el exceso de elementos puede estar limitando en forma solapada la producción en algunos establecimientos ganaderos, a tal punto se hace difícil que este problema sea reconocido por el productor como causa principal de la baja producción, sin embargo, en algunos casos es así. En los sistemas extensivos con reducido o nulo asesoramiento técnico existen otros factores productivos negativos que ocultan los efectos de las deficiencias 0 excesos de minerales (Bavera, 2000).

En las vacas lactantes, los macro minerales de principal importancia son cloruro de sodio $(\mathrm{NaCl})$, calcio $(\mathrm{Ca})$, fósforo $(\mathrm{P})$, y a veces magnesio $(\mathrm{Mg})$ y azufre (S) (Wattiaux y Howard, 1996). La fiebre de leche en los primeros días de la lactancia se debe a un desequilibrio en el metabolismo del calcio. El 
fósforo es esencial para mantener una buena fertilidad en el hato, casi todos los alimentos, con excepción de urea y grasa, contienen al mínimo cantidades limitadas de minerales. Debido a que las leguminosas contienen más calcio que las gramíneas, las raciones basadas en leguminosas requieren menos suplementación con calcio, la melaza es rica en calcio y los subproductos de origen animal son buenas fuentes de calcio y fósforo. El cloruro de sodio es el único mineral que se puede ofrecer ad-libitum o en bloques) (Wattiaux y Howard, 1996).

Comparando las fuentes de calcio y fósforo analizadas, se observa que el mayor aporte de fósforo lo suministra el biofos, luego otros fosfatos como el tricalfos y por último las harinas de hueso calcinada y vaporizada. Por lo tanto, el valor nutricional de biofos es superior debido al contenido más elevado de fósforo, a su aporte de calcio, a la mayor disponibilidad biológica, y a su estabilidad en cuanto a cambios en color, olor, sabor y textura. Se agrega a lo anterior que el fósforo es más limitante que el calcio en cuanto a disponibilidad comercial (Cardona et al., 2002) (Tabla 5).

Tabla 5. Composición nutricional promedia de fuentes minerales

\begin{tabular}{lccccc}
\hline Fuente de minerales & $\begin{array}{c}\text { Humedad } \\
(\%)\end{array}$ & $\begin{array}{c}\text { Proteína } \\
\text { cruda (\%) }\end{array}$ & $\begin{array}{c}\text { Cenizas } \\
(\%)\end{array}$ & Ca (\%) & P (\%) \\
\hline Biofos & 2 & - & - & 16,6 & 20,7 \\
Calcio, Carbonato & 0,21 & - & - & 38,8 & - \\
Fosfatos & 2,7 & - & - & 20,6 & 20 \\
Hueso, Calcinado & 0,6 & - & - & 35,7 & 15,6 \\
Hueso, Harina & - & 25,4 & 63 & 23 & 10,6 \\
Tricalfos & - & - & - & 32 & 18 \\
\hline
\end{tabular}

La suplementación mineral de la dieta de la vaca lechera es usualmente entre 0 y $150 \mathrm{~g} / \mathrm{vaca} / \mathrm{día}$ (Wattiaux, 1996), en la Tabla 6 se identifican los requerimientos de $\mathrm{Ca}$ y $\mathrm{P}$, en bovinos de lechería. Una mezcla de minerales que contiene calcio, fósforo o ambos (por ejemplo, fosfato dicálcico) puede ser requerida según los ingredientes de la ración. Los forrajes verdes usualmente contienen bajos niveles de fósforo en relación a las necesidades de la vaca. El ensilaje de maíz contiene poco calcio y fósforo, además requiere suplementación con ambos minerales. Los microminerales son requeridos en 
cantidades muy pequeñas y usualmente son incluidos como un premezclado en el concentrado (Wattiaux, 1996).

Tabla 6. Requerimientos diarios de calcio $(\mathrm{Ca})$ y fósforo $(\mathrm{P})$ para bovinos de leche

\begin{tabular}{|c|c|c|c|c|}
\hline \multirow{2}{*}{ Peso vivo (kg) } & \multirow{2}{*}{$\begin{array}{c}\text { Ganancia } \\
\text { (g/día) }\end{array}$} & \multirow{2}{*}{$\begin{array}{l}\text { Producción de } \\
\text { leche * (L/día) }\end{array}$} & \multicolumn{2}{|c|}{ Requerimientos (g/día) } \\
\hline & & & $\mathrm{Ca}$ & $\mathbf{P}$ \\
\hline 40 & 0.3 & -- & 6.8 & 4.1 \\
\hline 70 & 0.7 & -- & 15.4 & 7.7 \\
\hline 140 & 0.7 & -- & 19.4 & 11.4 \\
\hline 320 & 0.7 & -- & 24.9 & 18.6 \\
\hline 410 & 0.7 & -- & 28.6 & 20.9 \\
\hline 640 (seca) & -- & -- & 25.9 & 18.2 \\
\hline $640\left(\right.$ seca**$\left.^{* *}\right)$ & -- & -- & 41.8 & 25.4 \\
\hline 640 & -- & 16 & 73.5 & 46.7 \\
\hline 640 & -- & 25 & 102.5 & 65.0 \\
\hline 640 & -- & 33 & 121.1 & 75.3 \\
\hline
\end{tabular}

${ }^{*}$ leche al $3.5 \%$ de grasa butirosa. Se calcula $0.4-0.43 \%$ del consumo de MS.

** Último mes de seca antes del parto.

Modificado de Vieyra, (2004).

La desnutrición es el factor que más incide en la producción ganadera, especialmente en los países tropicales, las deficiencias y desequilibrios de minerales en el suelo y el forraje han sido considerados causantes de los problemas de baja producción y reproducción en el ganado de leche y/o carne. Investigaciones realizadas en regiones tropicales han señalado que la suplementación mineral puede resultar en aumentos de 20 a 100\% en las tasas de natalidad, además de una reducción significativa de la mortalidad (Laredo et al., 1987).

\section{LOS MINERALES EN LA REPRODUCCIÓN, SITUACIÓN EN LA GANADERÍA DE LECHERÍA}

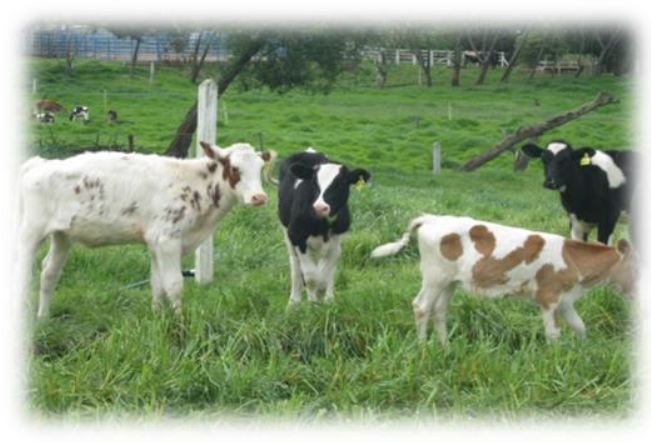

Para el normal crecimiento y futura producción de leche, las terneras deben recibir los minerales requeridos.

(Foto, maria ligia roa vega) 
Los minerales desarrollan un papel importante en la reproducción bovina, en algunas regiones de Colombia las deficiencias de minerales en sus suelos Para los bovinos, se han identificado aproximadamente 15 minerales esenciales y deben ser suministrados constantemente en forma adecuada para evitar deficiencias o excesos que puedan ocasionar problemas (Forero, 2004), dentro de éstos existen: Siete macro-minerales: Calcio (Ca), Fósforo (F), Potasio(K), Sodio (Na), Cloro (Cl), Magnesio (Mg), y Azufre (S), y ocho microminerales: Cobalto(Co), Cobre (Cu), Yodo (I), Hierro(Fe), Manganeso(Mn), Molibdeno (Mo), Selenio (Se) y Zinc(Zn) (Fader et al., 1995). Las deficiencias de macrominerales son raras debido a que la mayoría de los pastos reciben un aporte importante de ellos cuando se realizan fertilizaciones correctivas, situación que difícilmente se aplica a los microminerales pues su disponibilidad varía ampliamente y depende de condiciones muy específicas de $\mathrm{pH}$, salinidad, aireación, presencia de otros minerales y otros factores (Forero, 2004) (Tabla 7).

Tabla 7. Algunos minerales y sus principales acciones sobre el comportamiento reproductivo en bovinos

\begin{tabular}{|c|c|c|c|c|c|}
\hline \multirow{2}{*}{ Acción sobre la reproducción en bovinos } & \multicolumn{5}{|c|}{ Mineral responsable } \\
\hline & Mn & Zn & I & $\mathrm{Se}$ & $\mathbf{P}$ \\
\hline Disminuye la presentación de ovarios estáticos & $\mathrm{X}$ & $X$ & & & $\mathrm{X}$ \\
\hline Reduce el tiempo de inicio de la pubertad & $\mathrm{X}$ & $X$ & $x$ & & $\mathrm{X}$ \\
\hline Favorece el proceso de ovulación & $x$ & $X$ & & $\mathrm{X}$ & \\
\hline $\begin{array}{l}\text { Disminuye la presentación de anormalidades } \\
\text { fetales }\end{array}$ & $\mathrm{X}$ & $X$ & & & \\
\hline Favorece el incremento en tasas de concepción & $\mathrm{X}$ & & & $X$ & $\mathrm{X}$ \\
\hline Reduce la probabilidad de ovarios quísticos & $x$ & & & & $\mathrm{X}$ \\
\hline $\begin{array}{l}\text { Favorece la presentación de ciclos estrales } \\
\text { regulares }\end{array}$ & $\mathrm{X}$ & & & & \\
\hline Puede reducir la presentación de anestro & $X$ & & $\mathrm{X}$ & & \\
\hline Reduce la probabilidad de aborto & $\mathrm{X}$ & & $X$ & $X$ & \\
\hline Reduce la probabilidad de membranas fetales & & & $\mathrm{X}$ & $X$ & \\
\hline Ayuda en el crecimiento y desarrollo & $\mathrm{X}$ & & $\mathrm{X}$ & & $\mathrm{X}$ \\
\hline Favorece la resolución de casos de metritis & & & $X$ & $X$ & \\
\hline
\end{tabular}

Modificado de Forero, (2004).

Las actividades fisiológicas asociadas a la reproducción como presencia de ciclos estrales, gestación, lactación y crecimiento son exigentes desde el punto de vista mineral y requieren un suministro constante y adecuado de los 
mismos. Así, estos procesos establecen la necesidad de cuantificar los minerales requeridos ya que condiciones de subnutrición afectan considerablemente los procesos reproductivos del animal (Garmendia, 2010). Por ello los requerimientos de algunos minerales esenciales en el proceso reproductivo como calcio, fósforo, magnesio, cobre y zinc aportados por el suelo, las pasturas y la suplementación mineral durante el preparto y durante la lactación deben ser constantes y en cantidades necesarias (Tabla 8) (Garmendia, 2010; Brem et al., 2003).

Tabla 8. Concentración de minerales séricos relacionados a la etapa del ciclo estral

\begin{tabular}{|c|c|c|c|c|}
\hline PARÁMETRO & Metaestro & Diestro & Proestro-estro & Anestro \\
\hline Cobre (ug/dl) & $156,5 \pm 26,3$ & $180,3 \pm 35,5$ & $154,3 \pm 33,1$ & $167,2 \pm 46,3$ \\
\hline Calcio (mg/dl) & $10,40 \pm 1,67$ & $10,73 \pm 1,51$ & $11,52 \pm 1,37$ & $11,60 \pm 0,53$ \\
\hline Fósforo Inorg.(mg/dl) & $5,78 \pm 1,13$ & $5,74 \pm 0,74$ & $6,23 \pm 0,84$ & $6,62 \pm 0,62$ \\
\hline Magnesio (mg/dl) & $2,38 \pm 0,15$ & $2,35 \pm 0,22$ & $2,41 \pm 0,24$ & $2,33 \pm 0,10$ \\
\hline Hierro (ug/dl) & $143,0 \pm 33,4$ & $148,3 \pm 34,6$ & $149,5 \pm 35,3$ & $146,7 \pm 52,3$ \\
\hline
\end{tabular}

\section{PRINCIPALES ENFERMEDADES METABÓLICAS RELACIONADAS CON LOS MINERALES}

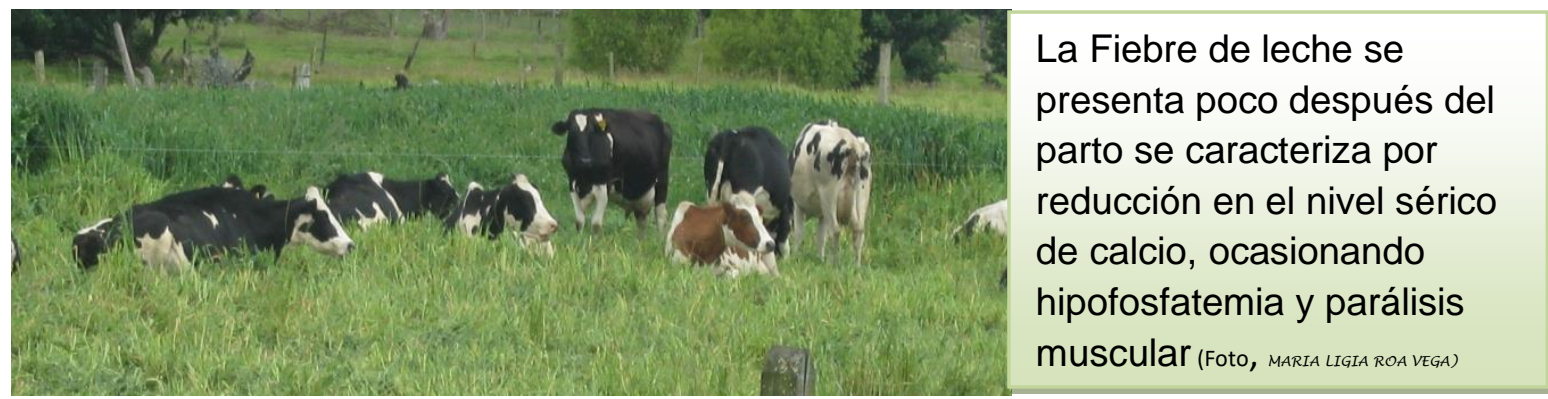

Entre las enfermedades metabólicas asociadas con desequilibrios minerales se debe tener en cuenta la hipocalcemia, mejor conocida como paresia puerperal y denominada entre los productores como fiebre de leche, este problema se presenta poco después del parto y es caracterizado por una baja en el nivel sérico de calcio en el animal, hipofosfatemia y parálisis muscular, en algunos de los casos más graves, la muerte del animal (Hove, 1986). Ocurre cuando falla la homeostasis del calcio, y su nivel sanguíneo que normalmente se encuentra se entre 9 y $10 \mathrm{mg} / \mathrm{dl}$ disminuye sus concentraciones por debajo de $5 \mathrm{mg} / \mathrm{dl}$ debido al drenaje de calcio exigido por la lactancia, afectando la función 
de músculos y nervios a tal punto que la vaca es incapaz de levantarse (Andresen, 2001).

En Colombia existen áreas deficientes en fósforo, su ingestión insuficiente se relaciona con problemas de baja fertilidad debido a una aparente disfunción ovárica determinando la disminución, inhibición o irregularidad en la presentación del celo (León et al., 2008), observándose en vacas de lechería, que la disminución en el consumo de este mineral provoca una baja en la producción de leche.

De otra parte, la concentración foliar de magnesio en los pastos de zonas lecheras en Colombia se encuentra en bajos niveles (Ceballos et al., 2004). Un aporte insuficiente unido a las perdidas por producción de leche, la interacción antagónica con otros minerales y por la presencia de factores que interfieren su absorción, puede causar deficiencias de magnesio especialmente en el inicio de la lactancia (Sandoval et al., 1998).

\section{ANÁLISIS, DISCUSIÓN Y PROBLEMÁTICA DE LA NUTRICIÓN MINERAL EN VACAS LECHERAS}

La utilización de minerales en la alimentación animal, contribuye a la disminución de la presentación de patologías que pueden estar relacionadas con el metabolismo general del animal o específicamente en sistemas como el reproductivo. La implementación de las dietas minerales depende de las eficiencias que presente el suelo y los pastos en los que se alimentan los animales.

Esta característica hace indispensable que cada producción realice los análisis necesarios y de esta manera se determinen las deficiencias o excesos de minerales a los que está expuesto el animal. La administración de sales mineralizadas de tipo comercial tiene que ser realizada bajo análisis técnicos los cuales arrojen como resultado la fórmula ideal para cada terreno, contribuyendo a la disminución de gastos en la producción suministrando a los animales exactamente lo que ellos requieren.

La solución de los problemas señalados promueve la adopción de arreglos tecnológicos, los cuales incluyen la búsqueda de alternativas en el manejo de 
las pasturas tendientes a optimizar la cantidad y calidad nutritiva del forraje, así como el uso de suplementos que potencien la eficiencia del uso de forraje o corrijan condiciones deficitarias para reducir la caída de la productividad durante las estaciones del año de acuerdo a la zona y las etapas fisiológicas críticas de los animales (Depablos et al., 2009). Las sales mineralizadas y los bloques multinutricionales son una opción válida para el control de estos problemas, sin embrago es importante tener en cuenta que requieren de un manejo apropiado en el hato, el cual implica la construcción de saladeros que contribuyan a disminuir el deterioro de la sal y reducir los costos por desperdicio.

\section{CONCLUSIONES}

La forma más práctica de garantizar un comportamiento reproductivo adecuado de las hembras, es garantizarles una alimentación adecuada en el periodo correspondiente al preparto y postparto, de esta manera impedir cambios de peso y condición corporal además de la presentación de enfermedades metabólicas (Garmendia, 2010).

En forma general, los suelos colombianos se han clasificado taxonómicamente como oxisoles y ultisoles ( $75 \%$ del área total), categorías que reúnen aquellos suelos de baja fertilidad actual y potencial, con niveles deficientes de macro y micro minerales ( $\mathrm{N}, \mathrm{P}, \mathrm{Ca}, \mathrm{K}, \mathrm{Mg}, \mathrm{Mn}$ y $\mathrm{Zn}$ ) y con gran concentración de aluminio (Forero, 2004). Estas características favorecen la creación de un ambiente denominado de acidez que se potencializa por la presencia excesiva de aluminio y baja proporción de otros minerales.

La acidez es la característica que determina la "biodisponibilidad de los minerales en el suelo y por tanto la que regula el valor nutricional de los pastos (Malagón, 2003). En nuestro medio, el pH de los suelos varía desde una categoría "fuertemente ácida" (5.6 a 6.0) hasta "extremadamente ácido" (< 4.5), lo que desde el punto de vista práctico se traduce en: fertilidad moderada a muy baja, materia orgánica reducida, pobre respuesta a fertilizantes o alcalinizantes y bajo potencial productivo (IGAC, 2003). 
Cuando se trata de macrominerales $(\mathrm{Ca}, \mathrm{P}, \mathrm{Mg})$, las deficiencias son fácilmente compensadas con la fertilización de los potreros y la suplementación con sales mineralizadas para consumo ad libitum. Sin embargo, tratándose de microminerales, los desbalances no son fácilmente detectables, pero sus implicaciones tanto productivas como reproductivas pueden considerarse económicamente importantes. Las demandas de microminerales en los animales varían constantemente y aumentan en forma significativa en estados de exigencias productivas y metabólicas como la preñez, la lactancia, época reproductiva, madurez sexual, crecimiento y desarrollo (Forero, 2004).

Las sales suministradas a los animales deben estar preparadas de acuerdo con las condiciones de cada terreno teniendo en cuenta los análisis bromatológicos, foliares, de suelos y de aguas, los cuales ofrecerán la información pertinente para la preparación de la sal y las proporciones de los minerales contenidas en ella. El suministro de las sales requiere de saleros cubiertos con acceso a la totalidad de los animales, que permitan mejorar la calidad de la sal suministrada al animal y disminuyan el desperdicio, reduciendo costos de producción.

\section{BIBLIOGRAFÍA}

1. Álvarez A, Lascano CE. Valor nutritivo de la sabana bien drenada de los Llanos Orientales de Colombia. Pasturas Tropicales (CIAT). 1987: 9 (3): 9-17.

2. Andresen HS. Vacas secas y en transición, Rev Inv Vet Perú. 2001: 12 (2): 36-48

3. Balbuena O, Luciani CA, McDowell LR, Conrad JH, Martin FG. Estudios de la Nutrición Mineral de los Bovinos para carne del este de las provincias de Chaco y Formosa (Argentina). Fósforo y Calcio. Instituto Nacional de Tecnología Agropecuaria. 2003.

4. Balocchi LO, Pulido FR, Fernández JV. Comportamiento de vacas lecheras en pastoreo con y sin suplementación con concentrado. Agricultura Técnica (Chile). 2002: 61 (1): $87-$ 98.

5. Brem JJ, Mestre J, Trulls HE, Pochon DO. Concentración sérica de minerales con relación al ciclo estral en bovinos Brangus. Rev Vet 2003: 14: 1.

6. Bavera GA. 2000. Suplementación mineral del bovino a pastoreo y referencias en engorde a corral, Capítulo 5. Ed. del autor, Río Cuarto. 103-108. Sitio Argentino de Producción Animal. [Consultado 04-06-10]. Disponible: www.produccion-animal.com.ar

7. Cardona M.G, Sorza JD, Posada SL, Carmona JC. Establecimiento de una base de datos para la elaboración de tablas de contenido nutricional de alimentos para animales. Rev Col Cienc Pec. 2002: 15: 2.

8. Ceballos A, Villa NA, Betancourth TE, Roncancio DV. Determinación de la concentración de calcio, fósforo y magnesio en el periparto de vacas lecheras en Manizales, Colombia. Rev Col Cienc Pec. 2004: 17:2.

9. Depablos L, Ordóñez J, Godoy S, Chicco, CF. Suplementación mineral proteica de novillas a pastoreo en los Llanos Centrales de Venezuela. Zootecnia Trop. 2009: 27(3): 249-262.

10. Fader OW, Marro O. Efecto de los minerales en la nutrición y salud animal en la región central de la provincia de Córdoba. INTA RAFAELA. Argentina, 1995. 
11. Forero LE. Fallas reproductivas asociadas a deficiencias de microminerales: caso colombiano. Universidad Nacional de Colombia. Dirección Científica Laboratorios Provet S.A. 2004 [Consultado 04-06-10]. www.produccion-animal.com.ar

12. Garmendia J. Los Minerales en la reproducción bovina. Facultad de Ciencias Veterinarias, Universidad Central de Venezuela, Maracay. 2010. [Consultado 04-06-10]. [Consultado 04-06-10]. http://avpa.ula.ve/docuPDFs/xcongreso/minerales.pdf

13. Gómez RG. Enciclopedia Bovina Universidad Nacional Autónoma de México. Facultad de Medicina Veterinaria y Zootecnia. Ciudad Universitaria. México, DF. 2008.

14. Gómez $C$ y Fernández M. Minerales para mejorar producción de leche y fertilidad en vacas lecheras. Departamento de Nutrición, Universidad Nacional Agraria La Molina, 2009. [Consultado 19-05-10]. Disponible en: http://www.infolactea.wordpress.com/2009/05/19/minerales-para-mejorar-produccion-deleche-y-fertilidad-en-vacas-lecheras-4/>

15. Hove $\mathrm{K}$, Cyclic changes in plasma and the calcium homeostatic endocrine system of the post parturient dairy cow. J. Dairy Sci; 1986; 69: 2072-2081.

16. Instituto Geográfico Agustín Codazzi. (IGAC). Mapa suelos de Colombia. Escala 1:500.000. Memoria explicativa. [En CD-ROM]. IGAC. Bogotá. 2003. [Consultado 04-0610]. Disponible: www.igac.gov.co.

17. Jaramillo, DA. El recurso suelo el recurso suelo y la competitividad del sector agrario colombiano. Universidad nacional de Colombia Medellín, 2004.

18. León JM, Mojica JE, Castro E, Cárdenas EA, Pabón ML, Carulla JE. Balance de nitrógeno y fósforo de vacas lecheras en pastoreo con diferentes ofertas de kikuyo (Pennisetum clandestinum) suplementadas con ensilaje de avena (Avena sativa). Rev Colomb Cienc Pecu; 2008: 21: 559-570.

19. Laredo MA, González CF, Huertas HB, McDowell LR. Los minerales y la producción de ganado de carne en pie de monte llanero. Zootecnia Tropical, 5 (1 y 2): 11-26. Sección Programa de Nutrición Animal ICA, El dorado, Bogotá, Colombia. La Libertad, Villavicencio, Colombia. 1987. [Consultado 04-06-10]. Sitio argentino de Producción Animal: www.produccion-animal.com.ar

20. Malagón D, Pulido C, Llinás R, Chamorro C. Suelos de Colombia: Origen, evolución, clasificación, distribución y uso. IGAC. Bogotá. 1995:632.

21. Malagón DC. Ensayo sobre tipología de suelos colombianos. Énfasis en génesis y aspectos ambientales- Rev. Acad. Colomb. Cienc. $2003: 27$ (104): 319-341.

22. Otálora PC, Garzón GA, Barrera GP. Frecuencia del polimorfismo lactoglobulina en una población de ganado Holstein de la Sabana de Bogotá. Corpoica Cienc. Tecnol. Agropecu. 2009: 10 (2): 191-195

23. Sandoval GL, Dellamea S, Pochon DO, Campos MV. Calcio, fósforo, magnesio y fosfatasa alcalina en vacas lecheras de una región subtropical suplementadas con óxido de magnesio. Rev Vet Méx; 1998: 29: 131-36.

24. Sans JI, Zeige, RS, Sarkarung S, Molina DL, Rivera M. Sistemas mejorados arrozpasturas para sabana nativa y pasturas degradadas en suelos ácidos de América del Sur. Centro Internacional de Agricultura Tropical (CIAT), Cali y Empresa Brasilera de Pesquisa Agropecuaria (Embrapa). 1999: 232-244.

25. Soil Survey Staff, (SSS), 1999. Soil Taxonomy. A basic system of soil classification for making and interpreting soil surveys. $2^{\mathrm{d}}$ ed. Agriculture Handbook № 436 . Soil Survey Staff. Washington D. C. 1999: 869.

26. Vieyra JM. El fósforo en la vaca lechera. Argent Export S.A. 2004. [Consultado 04-06-10]. Sitio argentino de Producción Animal. Disponible: www.produccion-animal.com.ar

27. Wattiaux MA. Reproducción y nutrición. Instituto Babcock para la investigación y desarrollo Internacional de la Industria Lechera, Universidad de Wisconsin-Madison, 1995.

28. Wattiaux MA, Howard WT. Guía técnica lechera: Alimentos para vacas lecheras. Instituto Babcock. Universidad de Wisconsin-Madison. USA. 1996: 24.

29. Wattiaux MA. Guía técnica lechera: reproducción y nutrición. Instituto Babcock. Universidad de Wisconsin-Madison. USA. 1996: 11. 